Objectives To enumerate and characterize HR-AKI cases among U.S. workers in a range of industries.

Methods Two data sources were analyzed: (1) archived case files of the Occupational Safety and Health Administration's (OSHA) Directorate of Technical Support and Emergency Management's Office of Occupational Medicine and Nursing from 2010 through 2020, and (2) the Severe Injury Reports database of work-related hospitalizations that employers reported to OSHA as required by regulation from 2015 to 2020. Confirmed cases of HR-AKI were ascertained by serum creatinine measurements. When creatinine measurements were unavailable, probable and possible cases of HR-AKI were ascertained from narrative incident descriptions. Industry-specific incidence rates of HR-AKI were computed. Capturerecapture methods assessed underreporting.

Results There were 607 cases of HR-AKI, including 22 confirmed cases and 585 probable or possible cases. HR-AKI occurred in a variety of indoor and outdoor industries including manufacturing, construction, mail and package delivery, and solid waste collection. Among the confirmed cases, 95.2\% were male, $50.0 \%$ had hypertension, and $40.9 \%$ were newly hired workers. Incidence rates from 1.0 to $2.5 \mathrm{HR}$-AKI hospitalizations per 100,000 workers per year were observed in high-risk industries. The capture-recapture analysis suggested that employers accurately reported only $41.2 \%$ of eligible HRAKI hospitalizations.

Conclusions Workers experienced HR-AKI in a diverse range of industries, including indoor facilities. Because of underreporting, data on work-related hospitalizations likely underestimate the true burden of occupational HR-AKI. Clinicians should be aware of kidney risk from recurrent heat stress. Employers should provide interventions to prevent kidney injury in heat-exposed workers.

\section{0-163 PROTECTION, RESILIENCE, EFFICIENCY AND PREVENTION (PREP), PREPARING WORKERS AND EMPLOYERS FOR A CHANGING CLIMATE}

'Jason Glaser, Erik Hanson, Kristina Jakobbson, Vidhya Venugopal, Esteban Arias, Heath Prince, Rebekah Lucas, Ilana Weiss, David H Wegman, Catharina Wesseling. 'La Isla Network, United States

\subsection{6/OEM-2021-EPI.55}

Introduction Chronic kidney disease of nontraditional cause (CKDnt), is a risk for millions of workers in Latin America and Asia. Treatment is expensive, resulting in early death for those affected. Strenuous work in extreme heat without sufficient rest and hydration is an important driver. Without prevention, this epidemic will likely accelerate due to climate change.

Objectives We established the Adelante Initiative, a workplace intervention focusing on heat stress mitigation, together with improved ergonomics, and organizational assessment designed to prevent CKDnt among workers at a sugarcane mill in Nicaragua. We aim to adapt the program to other geographies and industries.

Methods PREP builds on Adelante, investigating the following: 1)immediate and long-term impact of the intervention has on workforce health and productivity; 2)economic and social impacts on those affected by CKDnt and whether intervention promotes resilience 3)the economic burden on health systems treating CKDnt; 4) analysis of public and private policies to understand what policy factors (present or absent), have contributed to the disease and what policies are required to address it.

Preliminary observations Despite the inconsistent implementation of intervention there is a $72 \%$ reduction of kidney injury. The economic burden in households with CKDnt is dire. CKDnt is depleting health systems. Current public and private policies are insufficient to address CKDnt though signs are encouraging within the development sector and some industries.

Conclusions Knowledge gained will create the groundwork to expand to other sugarcane mils and eventually other industries at-risk for heat stress and CKDnt. Climate change means more extreme temperatures in already impacted regions and the likelihood that regions further north and south of the equator will also be impacted by CKDnt. It is essential to develop a model to protect worker health and productivity. Occupational heat stress and resulting CKDnt require increased attention and resources.

\section{0-370 HOT AMBIENT TEMPERATURE AND ITS IMPACTS ON ADVERSE PREGNANCY OUTCOME (APO) -A SYSTEMATIC REVIEW}

${ }^{1}$ S Rekha, Vidhya Venogopal, SJ Nalini, S Bhuvana, S Kanmani. 'Sri Ramachandra Institute of Higher Education and Research, India

\subsection{6/OEM-2021-EPI.56}

Introduction Exposures to hot ambient temperature have been associated with pregnancy complications and congenital abnormalities among working pregnant women. Workplace heat is likely to exacerbate the Adverse Pregnancy Outcomes (APO) especially with rising temperatures in tropical countries. Research evidence that associates occupational heat stress and APO are scarce and there is a need for new types of knowledge in this research area.

Objective Review the evidence from published literature on the association between exposure to hot ambient temperature and APOs.

Methods We conducted a systematic literature review of the published literature on the effects of hot ambient/occupational temperature and its impacts on pregnancy outcomes from databases such as Pub Med, Cochrane, Web of Science, and Science direct. We searched more than 500 related original \& review articles, short communications, dissertations, newsletters, and book chapters. We systematically grouped the reviewed literature into the following categories: Heat stress/ strain, adverse maternal, fetal outcome, and interventions. The categorized literature was then analyzed and examined the principal findings of the studies.

Results This review found a convincing association between heat stress and APOs among the exposed pregnant women, including miscarriages, preterm birth, stillbirth, low birth weight (LBW), and congenital anomalies. Furthermore, few articles found occupational heat may intensify the complications, an anticipated risk, which may trigger APOs during all the trimesters. Our review informs the magnitude and significance of the impacts of heat stress on pregnant women's health, providing useful information for exploring the biological mechanisms of APOs and also outlines various interventions to prevent heat-related APOs.

Conclusions The limited quantity of existing review gave us key insights and reemphasized the need for more extensive 